1Dept of Clinical Medicine, Trinity Centre for Health Sciences, Multidisciplinary Intensive Care Research Organization (MICRO), Wellcome Trust, HRB Clinical Research,

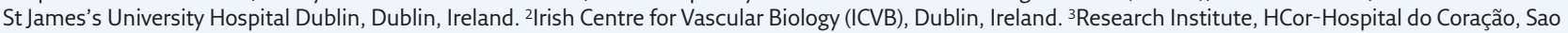
Paulo, Brazil. 4Polyvalent Intensive Care Unit, Hospital de São Francisco Xavier, Centro Hospitalar de Lisboa Ocidental, Lisbon, Portugal. 5 NOVA Medical School, CEDOC, New University of Lisbon, Lisbon, Portugal. ${ }^{6}$ Dept of Pulmonology, Hospital Clinic of Barcelona, IDIBAPS, CIBERES, Barcelona, Spain. ${ }^{7}$ Pulmonary Division, Heart Institute, Hospital das Clínicas, University of São Paulo, Sao Paulo, Brazil. ${ }^{8}$ Academic Medical Center, Amsterdam, The Netherlands. ${ }^{9}$ Dept of Pathophysiology and Transplantation, University of Milan, Cardio-thoracic unit and Adult Cystic Fibrosis Center, Fondazione IRCCS Cà Granda Ospedale Maggiore Policlinico, Milan, Italy.

\title{
Respiratory research networks in Europe and beyond: aims, achievements and aspirations for the 21st century
}

Healthcare-associated infection, such as intensive care unit (ICU)-related respiratory infections, remain the most frequently encountered morbidity of ICU admission, prolonging hospital stay and increasing mortality rates. The epidemiology of ICU-related respiratory infections, particularly nonventilated ICU-associated pneumonia and ventilator-associated tracheobronchitis, appears to be quite different among different countries. European countries have different prevalence, patterns and mechanism of resistance, as well as different treatments chosen by different attending physicians. The classical clinical research process in respiratory infections consists of the following loop: 1) identification of knowledge gaps; 2) systematic review and search for adequate answers; 3) generation of study hypotheses; 4) design of study protocols; 5) collection clinical data; 6) analysis and interpretation of the results; and 7) implementation of the results in clinical practice.

@ ERSpublications

Learn about ENIRRI, an @ERStalk Clinical Research Collaboration http://ow.ly/ct6m30dbXiP
Cite as: Martin-Loeches I, Zampieri F, Povoa P, et al. Respiratory research networks in Europe and beyond: aims, achievements and aspirations for the 21st century. Breathe 2017; 13: 209-215.
Healthcare-associated infection (HCAl) is a major healthcare problem [1]. HCAl, in the guise of intensive care unit (ICU)-related respiratory infections, remains the most frequently encountered morbidity of ICU admission, prolonging hospital stay and increasing mortality rates. ICUrelated respiratory infections (IRRIs) usually present as uncomplicated infections that may precipitate severe sepsis or septic shock [2]. In patients with established sepsis, IRRIs complicate recovery and lead to an increase in mortality rate [3].

Current IRRI control measures, comprising a series of protocol-driven physical interventions designed to limit cross-infection with multiresistant organisms, are not individualised and are only partially effective. The causative organisms in IRRI are common commensal organisms that are frequently antibiotic sensitive and arise in patients who should mount an effective adaptive immune response (figure 1). While IRRI risk factors are well described and most include demographic attributes, they are poorly sensitive as predictors of HCAl and are largely immutable [4].

Over the last few years, a significant number of randomised controlled trials (RCTs) have been conducted in order to determine the efficacy of new 


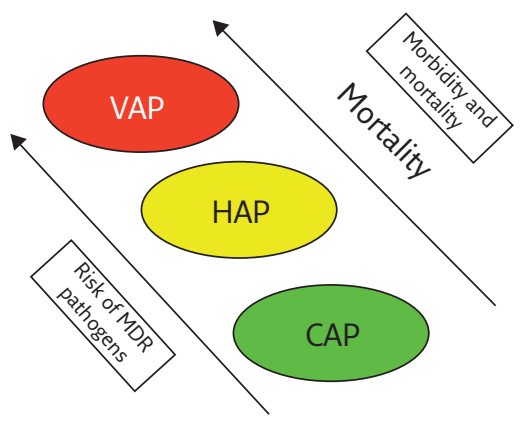

Figure 1 Landscape of respiratory infections. CAP: community-acquired pneumonia; HAP: hospital-acquired pneumonia; VAP: ventilator-associated pneumonia; MDR: multidrug-resistant.

drugs for multidrug-resistant (MDR) pathogens, namely Pseudomonas aeruginosa, Acinetobacter baumannii, methicillin-resistant Staphylococcus aureus, Klebsiella pneumoniae and carbapenemaseproducing bacteria. In Europe, research into respiratory infections in critical care lags behind that in the USA, where the 10×'20 Initiative has been set up with the aim to develop 10 new antibacterial drugs by 2020 . A joint commission of the US Centers for Disease Control and Prevention and the European Centre for Disease Prevention and Control developed a standardised international terminology to describe profiles of resistance, including MDR, extensively drug-resistant and pandrug resistant bacteria [2]. The emerging increase in antibiotic consumption and, therefore, resistances makes the importance of MDR especially relevant, leading to the implementation of antibiotic stewardship. Different antibiotics are currently

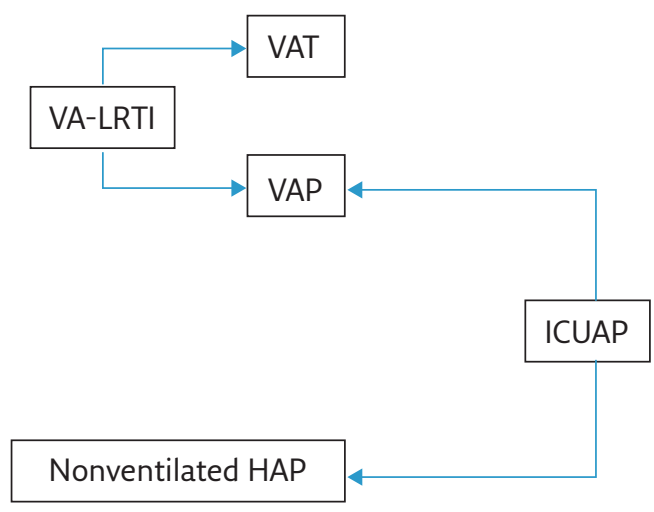

Severe non-ICU HAP

Ward

Figure 2 Scheme of ICUAP. VA-LRTI: ventilator-associated lower respiratory tract infection; MV: mechanical ventilation. under identification and development, many of which have new modes of action.

The epidemiology of IRRIs, particularly nonventilated ICU-associated pneumonia (ICUAP) and ventilator-associated tracheobronchitis (VAT), appears to be quite different among different countries (figure 2). The published literature is dominated by monocentric data and is biased by the local microbiology, limiting the generalisability of findings to different regions and countries worldwide. Therefore, there is a need to establish a research network across Europe that will foster interaction among researchers, physicians and industry.

In 2014, the European Respiratory Society (ERS) funded a new Clinical Research Collaboration, the European Network for ICU-Related Respiratory Infections (ENIRRI), with the aim to support a group of experienced researchers across Europe to answer important research questions in the field of epidemiology, aetiology, microbiology, pathophysiology, clinical management and prognosis of IRRI. ENIRRI currently includes representatives from eight European countries and 14 centres in Italy, Portugal, France, Spain, Greece, Germany, the Netherlands and Croatia. Each of these centres is a leader in their country or region and will be asked to involve additional local centres. In 2017, the network expanded to countries in Latin America, namely Brazil and Argentina. Members of ENIRRI have an extensive background in IRRIs, and will work together to perform clinical studies and promote educational activities in the field within the ERS (table 1).

Both clinical and translational research will be developed within ENIRRI. This will be facilitated by the development of a shared database of clinical data regarding IRRIs, including both epidemiological and outcome data. Translational studies using this platform may include the establishment of biobanks and the development of studies to investigate mechanistic research questions, including those on immunological or microbiological aspects. This might finally drive a change in clinical practice and an improvement in patient care.

\section{Design of studies}

ENIRRI will provide epidemiological data on the occurrence and outcomes of lower respiratory tract infections in critically ill patients. These epidemiological data are not limited to the incidence of the problem but also include detailed data on demographics, microbiological detection methods, causative agents, modality of respiratory support (supplementary oxygen, noninvasive ventilation, high-flow nasal cannulae and mechanical ventilation) and therapy, among others.

The most straightforward type of study will include simple descriptive analysis, which would represent an important starting point. Subsequent 
To create a global database of patients with IRRIs in order to facilitate epidemiological and clinical research, and recruitment into clinical trials

To attract new researchers into the IRRIs through presentations at the ERS International Congress, and dissemination of research priorities and knowledge

To support and encourage early career researchers, many of who are applicants in this proposal, through multidisciplinary training opportunities and participation at senior levels in network activities

To facilitate applications to industry and EU sources to build IRRI research capacity in Europe and beyond

To build a network of experts in IRRI management who will determine the need for a task force, and the need for an IRRI consensus statement or guidelines for diagnosis, prevention and management

EU: European Union.

studies will be used to assess the independent association between specific exposures/procedures and relevant clinical outcomes. Examples will be the assessment of the possible association between specific pathogens and mortality in ventilatorassociated pneumonia (VAP), or between timing of intubation in nosocomial pneumonia and outcome. In these situations, statistical methods for correcting for confounders (logistic regression, generalised additive models, etc.) and/or pairing methods (propensity matching and GenMatch) are useful, and provide relevant insights when trials are unfeasible. While residual confounding will remain even after the proper use of such analyses (as with any observational study), the results will be used as hypothesis generation for future RCTs (sample size estimation, for example). This, coupled with the network of devoted centres in ENIRRI, may result in focused RCTs that will take place in specific centres, where specific interventions may be most effective and needed. For instance, trials evaluating potential therapies for MDR bacteria will be run across those ICUs showing a high density of such pathogens while the rest of the network will take care of other research questions.

\section{Past success}

The classical clinical research process consists of the following loop:

1) identification of knowledge gaps

2) systematic review and search for adequate answers

3) generation of study hypotheses

4) design of study protocols

5) collection of clinical data

6) analysis and interpretation of the results

7) implementation of the results in clinical practice

At the end, the new changes in practice would generate new knowledge gaps and the loop starts again [5]. Any research process differs from the previous one in view of the important input coming from daily clinical practice at all stages of the research process [6].

In the early 1980s, clinicians from several backgrounds began to build networks to study important clinical questions (practice-based research networks), having recognised the limits of small sample sizes. These networks were constituted by groups of clinicians from different institutions (both academic and nonacademic) devoted to the care of patients and who were engaged in the treatment of particular diseases or conditions. This method allows the evaluation of whether a special intervention is safe, effective and sustainable in the real daily practice across different environments. Additionally, these networks that reflect daily clinical practice are a "real lab" to test different interventions and, finally, to perform translational medicine [7].

In the 1980s, two different networks in the area of cardiology produced two landmark clinical trials on the role of thrombolytic therapy with streptokinase in acute myocardial infarction (AMI) $[8,9]$ resulting from a collaboration of two Italian organisations, the Mario Negri Institute and the Associazione Nazionale dei Medici Cardiologi Ospedalieri (ANMCO). Mario Negri (1891-1960) was a Milanese jeweller and philanthropist who helped to create the Mario Negri Institute, a notfor-profit research organisation dedicated to helping defend human health and life. ANMCO is a nonprofit association with $>5000$ members, formed by Italian cardiologists working in the national health service. It was also founded in the 1960s with the aim of promoting good clinical practice, the prevention and rehabilitation of cardiovascular diseases through organisational proposals, vocational education and training, promotion and conducting of studies and research, and leadership in preparation and development of standards and guidelines. In the 1980s, these two organisations joined together, creating the Gruppo Italiano per lo Studio della Streptochinasi nell'Infarto (GISSI). GISSI conducted several landmark RCTs, GISSI-1 (streptokinase in AMI) [10], GISSI-2 (alteplase in AMI) [11], 
GISSI-3 (lisinopril and nitrates in AMI) [12] and GISSI-prevention (fatty acids and vitamin E) [13], that markedly impacted on the approach to AMI. In total, this network enrolled $>60000$ patients with $\mathrm{AMI}$ across these four RCTs, leading to a change in the approach to AMI, in terms not only of therapy (thrombolysis) but also of early recognition and intervention, with a marked decline in $\mathrm{AMI}$ mortality. This represents a classic example of practicebased research networks, involving academic and nonacademic institutions, but others were also implemented almost simultaneously among general practitioners, namely the Ambulatory Sentinel Practice Network and Dartmouth Cooperative Information Project (COOP). Unfortunately, on average, it took 17 years for $14 \%$ of new discoveries to enter day-to-day clinical practice [7, 14].

\section{Difficulties for networks}

Building up clinical research networks faces several difficulties and constrains that can be divided into technical, legal and human or organisational factors [5]. The most difficult step to achieve stems from the need for a dedicated team to coordinate and support the network along with the importance of having a research nurse. The implementation of a clinical research network needs to have a common information system that receive and collect all the clinical data from different members/institutions across the network [15]. To accomplish this task, the information system of the network should be able to communicate with the information technologies of different members/institutions (interoperability) and in addition, should also support and manage big and complex data. The problem of difficult interoperability is also a consequence of other factors. These informatic solutions have different architectures and languages, use different medical terminologies and clinical definitions, and address different problems in different institutions, which altogether creates big challenges for the implementation of a network, and some institutions still have limited electronic records, making data migration even more difficult.

Data protection is also an issue that might raise ethical and legal problems. When individual medical records are used for research purposes, this must to comply with the national regulations and requirements of the local institutional review board (IRB). The law differs, sometimes markedly, from country to country, namely concerning individual data protection. Besides this, even the local IRBs sometimes have different interpretations of the legal framework. These are frequent constraints that could create difficulties in the implementation of the network.

Finally the human and organisational factors are also a frequent problem. Usually, the networks result from different collaborations: university, hospitals, primary care, industry and research laboratories [16]. Regardless of the type of clinical research network, a fundamental issue is building a strong trust between these partners, and a perfect knowledge of its structure, organisation and steering committee is needed. To accomplish this, frequent, open and honest communication is needed to respect different cultures and clinical expectations. Another crucial aspect to keep the network active is to ensure to all partners mutual benefits from their individual participation.

A final constrain of a clinical research network is funding. These organisations need constant funding to preserve the network and its maintenance. This is frequently difficult to achieve, and these networks frequently face large fluctuations and difficulties in funding that challenges its existence.

\section{Consortium of networks and industry}

In recent years, there has been an expansion in the aims of research networks. Indeed, networks are currently aiming not only to conduct highquality research but also to promote advocacy and integrate patients, their relatives and nonprofit organisations, such as disease associations. As part of the networks' expansion, an active and potentially productive partnership between the networks and industry has emerged. By constructing consortia, some networks could draw on expertise from industry regarding new technology incorporation, business, highly organised structure and funding. Importantly, the contracts between these networks, both academic and nonacademic, and industry have been extensively debated, and clear concerns have emerged over potential conflicts of interest, authorship, copyright, and other interests for safeguarding ethics and patients' interests. These partnerships have provided sustainability in terms of funding, high recruitment rates and sharing of knowledge. Similarly, opportunities have arisen for academic educational grants and formal training (e.g. PhD or postdoctoral positions) integrating industry-based periods, and student involvement outside academia. Another important point regarding consortia regards the fact that funding usually comes partly from governmental or nonprofit organisations and partly from industry. We need time to evaluate if the current model of partnership, with shared funding and methods of organisation, will be sustainable, efficient and accepted in the community. A great example of the aforementioned partnerships that has been highly productive in the respiratory disease field is EMBARC, the European Multicentre Bronchiectasis Audit and Research Collaboration [17].

\section{European perspective}

The European community is a multicultural and rich environment for collaborative research networks. Indeed, it has great possibility to 
incorporate different socioeconomic levels, genetics, human behaviours, and nutritional and health interventions with the availability of common funding agencies, such as the European Commission, and previous academic successful partnerships and medical societies as hubs for propagating the network. However, it is well known that some barriers are still present. Networks have faced problems with investigator communication, mainly because English proficiency may be not truly sufficient to facilitate a networking engagement with some countries or, for instance, community centres. Additionally, each country has specific laws for research regarding not only ethics approval but also tax exemption programmes for academic institutions. Networks will need to create new tools and apply different approaches to overcome these barriers. Otherwise, it is likely that some European countries will be underrepresented in network databases. Some barriers are associated with country development and economy but not exclusively, and networks should share their experience to account for these disparities.

In respiratory medicine and associated areas, European networks have been successful and the diversity of the European population, prevalent diseases and health systems have led to hypothesis to be tested and standardisations, moving the respiratory field steps forward. Different exposure levels to air pollution and smoking, different prevalences of causes of structural lung diseases, and incidence of lung infections, such as tuberculosis, are strengths to conducting research in the respiratory field in Europe. ENIRRI also provides a fundamental basis to understand nosocomial infections across Europe. Hospital-acquired pneumonia is the most common nosocomial infection, and is associated with high costs, prolonged length-of-stay and mortality. One of the main aims of ENIRRI will be to investigate pneumonia caused by MDR pathogens. European countries have different prevalence, patterns and mechanism of resistance, as well as different treatments chosen by different attending physicians. ENIRRI will give a new and interesting perspective on what is ongoing in real life with antibiotic-resistant pathogens and their spread around Europe.

\section{Global perspective in middle-low-income countries}

Large heterogeneity is expected when different centres from different regions are included in a study. This heterogeneity is expected to dramatically increase when centres from different countries are included, which is true for both observational and interventional studies [18]. Regarding observational studies in critically ill patients, differences in local practices, microbiological patterns and ICU admission policies may play an extremely important role in determining heterogeneity. While heterogeneity may limit the extension of the observed data to other settings in the context of prediction, understanding factors associated with it may prove of greater value for both local and global practices, as mentioned earlier.

HCAls are an important health issue in middle-low-income countries (MLICs), where pneumonia is one of the most frequent sources of infection (up to 15\%) [19]. In this sense, the inclusion of MLIC in ENIRRI will contribute to understanding the factors associated with the outcome of lower respiratory tract infection in scenarios that are strikingly different from Europe (table 2). The microbiological profile is one of the key differences between MLICs and high-income countries (HICs). In LMICs, Gram-negative rods account for $>70 \%$ of VAPs, one quarter being caused by Acinetobacter species [19]. For certain pathogens occurring in LMICs, such as panresistant Klebsiella spp. (including stains resistant to polymyxin), there is not even consensus on what is the best treatment, representing a field in desperate need for research.

Data obtained through the ENIRRI network has the goal to provide robust and reliable data for clinical practice guidelines in many clinical scenarios, from cutting-edge units in HICs to resource-constrained units in LMICs.

\section{Conclusion}

HAP is the most frequent hospital-acquired infection in the ICU [1]. IRRIs are very common nowadays, with different mechanisms of acquisition. In addition, whilst mechanical ventilation is potentially lifesaving, it also carries significant risks and complications [2]. Of these, ICUAP is associated with significant morbidity, mortality and use of healthcare resources. More severe forms of ICUAP are when patients develop such infection under mechanical ventilation, which is called VAP; conversely, VAT is believed to be an intermediate stage between colonisation of the lower respiratory tract and VAP. However, more recent data suggest that VAT may be a separate entity that may contribute to increased length of ICU stay and longer duration of mechanical ventilation. Due to the heterogeneity of criteria for admission in ICU across Europe, the burden of respiratory infections in critically ill patients remains unknown. Moreover, the numbers of episodes of either VAT or ICUAP in ventilated and not ventilated patients, and a lack of evidence-based treatments, necessitate a coordinated approach to research and clinical care in different countries around the globe.

Several reports have been launched from individual data; however, no multidisciplinary 
Table 2 Sources of heterogeneity: LMIC particularities

\section{Baseline age, comorbidities and performance status}

Age, comorbidities and previous performance status are important determinants of critical care outcome

Baseline health status and comorbidities have a different profile in LMICs when compared to HICs [7, 8]

\section{ICU admission policies}

Patients may be more prone to be admitted to the ICU according to bed availability or admission policies

Paucity of ICU beds in Latin American and other LMICs may compromise appropriate timing for ICU admission and result in higher mortality $[9,10]$

\section{Local practices in mechanical ventilation}

Some units may choose to start mechanical ventilation early and/or may adopt early weaning protocols; thereby, duration of mechanical ventilation may vary

Ventilator settings may also be different (i.e. tidal volume)

Mortality of acute respiratory failure is high in LMICs and adherence to best practices may be lower $[11,12]$

\section{Diagnostic technique availability}

Diagnostic techniques may vary between centres and countries

LMICs may have restricted access to basic and advanced diagnostic methods [10]

\section{Microbiological profile}

Causative pathogen is associated with outcome in nosocomial pneumonia

Gram-negative rods (including MDR pathogens) are more common in LMICs, especially Latin America [13]

\section{Initial empirical antimicrobial therapy}

Lack of adherence to early antimicrobial therapy may impact clinical outcomes

Sepsis awareness is lower in LMICs [14]

\section{Use of prevention strategies}

Several prevention strategies may be effective in preventing nosocomial pneumonia, especially VAP (subglottic suctioning tubes, coated tubes, etc.)

Limited availability of some devices in LMICs

Lack of availability of single rooms in some settings [15]

\section{EOL preferences}

EOL policies vary between different countries and between different cultures

This may result in changes in outcome estimates

Physicians in Latin America may be less prone to establish clear EOL policies and to discuss treatment directives with patients and relatives [16]

HIC: high-income country; EOL: end-of-life.

networks for research in ICUAP in ventilated and not ventilated patients exist currently. This multidisciplinary Clinical Research Collaboration supported by the ERS will provide a forum to drive discovery at translational and clinical levels, and provide a platform for applications for network funding to the European Union and pharmaceutical industry. The goal of this collaboration is to promote clinically orientated research in the field of respiratory infections in critical care settings across Europe and beyond, by sharing ideas, research protocols and data between members of the network. There is a renewed interest in IRRIs from the pharmaceutical industry and from public funding bodies in order to determine the efficacy of new drugs for MDR bacteria. Research has been always a matter of connection. When we analyse the papers published in recent years, those that were more cited and that have impacted daily clinical practice are those that have been conducted under the development and framework of a research network. This concept gains a higher dimension when we analyse the impact of infectious diseases at the most severe end, i.e. critically ill patients. Physicians all over the world are especially sensitive to guideline recommendations in order to provide the best care for their patients. This approach is valid but nowadays the establishment of clinical networks helps to determine the impact of recommendations based on particular geographic settings. 


\section{Conflict of interest}

Disclosures can be found alongside this article at breathe.ersjournals.com

\section{References}

1. Zilahi G, Artigas A, Martin-Loeches I. What's new in multidrugresistant pathogens in the ICU? Ann Intensive Care 2016; 6: 96

2. Martin-Loeches I, Deja M, Koulenti D, et al. Potentially resistant microorganisms in intubated patients with hospital-acquired pneumonia: the interaction of ecology, shock and risk factors. Intensive Care Med 2013; 39: 672-681.

3. Martin-Loeches I, Levy MM, Artigas A. Management of severe sepsis: advances, challenges, and current status. Drug Des Devel Ther 2015; 9: 2079-2088.

4. Nseir S, Martin-Loeches I, Makris D, et al. Impact of appropriate antimicrobial treatment on transition from ventilatorassociated tracheobronchitis to ventilator-associated pneumonia. Crit Care 2014; 18: R129.

5. Williams RL, Johnson SB, Greene SM, et al. Signposts along the $\mathrm{NIH}$ roadmap for reengineering clinical research: lessons from the Clinical Research Networks initiative. Arch Intern Med 2008; 168: 1919-1925.

6. Green LA, Fryer GEJ, Yawn BP, et al. The ecology of medical care revisited. N Engl J Med 2001; 344: 2021-2025.

7. Westfall JM, Mold J, Fagnan L. Practice-based research: Blue Highways on the NIH roadmap. JAMA 2007; 297: 403-406.

8. Randomised trial of intravenous streptokinase, oral aspirin, both, or neither among 17,187 cases of suspected acute myocardial infarction: ISIS-2. Lancet 1988; 2: 349-360.

9. Effectiveness of intravenous thrombolytic treatment in acute myocardial infarction. Lancet 1986; 1: 397-402.

10. Long-term effects of intravenous thrombolysis in acute myocardial infarction: final report of the GISSI study. Lancet 1987; 2: 871-874.
11. GISSI-2: a factorial randomised trial of alteplase versus streptokinase and heparin versus no heparin among 12,490 patients with acute myocardial infarction. Lancet 1990; 336: 65-71.

12. GISSI-3: effects of lisinopril and transdermal glyceryl trinitrate singly and together on 6-week mortality and ventricular function after acute myocardial infarction. Lancet 1994; 343: 1115-1122.

13. Dietary supplementation with $n-3$ polyunsaturated fatty acids and vitamin $\mathrm{E}$ after myocardial infarction: results of the GISSI-Prevenzione trial. Lancet 1999; 354: 447-455.

14. McGlynn EA, Asch SM, Adams J, et al. The quality of health care delivered to adults in the United States. $N$ Engl J Med 2003; 348: 2635-2645.

15.Zerhouni EA. US biomedical research: basic, translational, and clinical sciences. JAMA 2005; 294: 1352-1358.

16. Rahman A. Why do collaborative research? BMJ 2007; 335: 304.

17. Chalmers JD, Aliberti S, Polverino E, et al. The EMBARC European Bronchiectasis Registry: protocol for an international observational study. ERJ Open Res 2016; 2: 00081-2015.

18. Marschner IC. Regional differences in multinational clinical trials: anticipating chance variation. Clin Trials 2010; 7: 147-156.

19. Allegranzi B, Bagheri Nejad S, Combescure C, et al. Burden of endemic health-care-associated infection in developing countries: systematic review and meta-analysis. Lancet 2011; 377: 228-241. 